Document downloaded from:

http://hdl.handle.net/10251/60687

This paper must be cited as:

González Albuixech, VF.; Giner Maravilla, E.; Tarancón Caro, JE.; Fuenmayor Fernández, FJ.; Gravouil, A. (2013). Convergence of domain integrals for stress intensity factor extraction in 2-D curved cracks problems with the extended finite element method. International Journal for Numerical Methods in Engineering. 94(8):740-757. doi:10.1002/nme.4478.

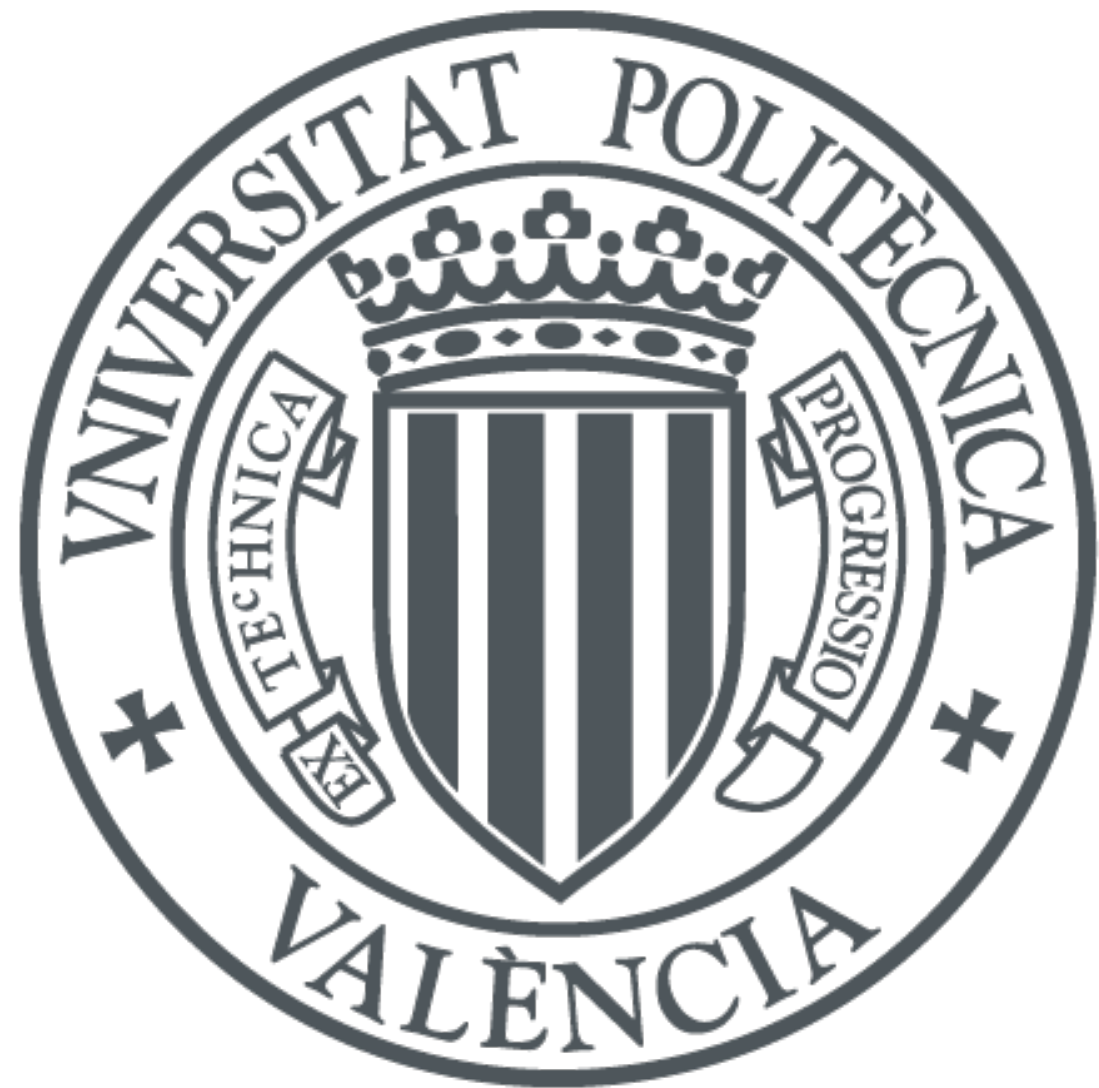

The final publication is available at

http://dx.doi.org/10.1002/nme.4478

Copyright Wiley

Additional Information 


\title{
Convergence of domain integrals for stress intensity factor extraction in 2-D curved cracks problems with the extended finite element method
}

\author{
V. F. González-Albuixech ${ }^{1 *}$, E. Giner ${ }^{1}$, J. E. Tarancón ${ }^{1}$, F. J. Fuenmayor ${ }^{1}$ and \\ A. Gravouil ${ }^{2}$ \\ ${ }^{1}$ Centro de Investigación de Tecnología de Vehículos - CITV. Dpto. de Ingeniería Mecánica y de Materiales. Universitat \\ Politècnica de València. Camino de Vera s/n, 46022 Valencia, Spain. \\ ${ }^{2}$ Laboratoire de Mécanique des Contacts et des Structures (LaMCoS). INSA-Lyon UMR CNRS 5259. 20 avenue Albert \\ Einstein, F-69621 Villeurbanne, France
}

\begin{abstract}
SUMMARY
The aim of this study is the analysis of the convergence rates achieved with domain energy integrals for the computation of the stress intensity factors (SIF) when solving 2-D curved crack problems with the extended finite element method (XFEM). Domain integrals, specially the $J$-integral and the interaction integral, are widely used for SIF extraction and provide high accurate estimations with finite element methods. The crack description in XFEM is usually realized using level sets. This allows to define a local basis associated with the crack geometry. In this work the effect of the level set local basis definition on the domain integral has been studied. The usual definition of the interaction integral involves hypotheses that are not fulfilled in generic curved crack problems and we introduce some modifications to improve the behavior in curved crack analyses. Despite the good accuracy of domain integrals, convergence rates are not always optimal and convergence to the exact solution cannot be assured for curved cracks. The lack of convergence is associated with the effect of the curvature on the definition of the auxiliary extraction fields. With our modified integral proposal, the optimal convergence rate is achieved by controlling the $q$-function and the size of the extraction domain.
\end{abstract}

KEY WORDS: SIF; curved cracks; domain integrals; interaction integral; $J$-integral; convergence rate; level set

\section{INTRODUCTION}

The analysis of the convergence rates achieved with different domain energy integrals and the corresponding stress intensity factors (SIFs) when solving problems with curved cracks with the extended finite element method (XFEM) $[1,2,3,4,5,6]$ is still an unresolved issue. The SIFs characterize the severity of a crack and therefore a good estimation of these factors is important. It is well known that domain integrals, based on the $J$-integral $[7,8]$ and the interaction

*Correspondence to: Centro de Investigación de Tecnología de Vehículos - CITV. Dpto. de Ingeniería Mecánica y de Materiales. Universitat Politècnica de València. Camino de Vera s/n, 46022 Valencia, Spain. E-mail: vigonal@upvnet.upv.es 
integral $[3,9,10,11]$ are widely used for SIF extraction and provide high accurate estimations with finite element methods. However, their application to curved cracks in combination with the XFEM involves the introduction of certain approximations that can have an influence on the SIF accuracy and convergence rate.

The XFEM and level set method (LSM) [12,13] can be used for the analysis of curved cracks in two dimensions and non-planar cracks in three dimensions [3, 4, 11]. However, it is necessary to understand the effect of the level set and the local basis definition on the SIF extraction using domain integrals for problems with curved cracks. In general, building orthogonal level set distance functions is not possible for curved cracks and therefore the analytically constructed curvilinear basis [3] does not fulfill the orthogonality condition. However, it is possible to define an orthogonal basis [11] using only the level set function whose zero value corresponds to the crack face, together with geometric considerations. Both choices are used in the bibliography, but the lack of a convergence study does not give an indication about which definition is better. The first goal of this work is to perform an error convergence study in a curved crack problem and to provide arguments to choose the basis.

Not all the domain integrals are suitable for the study of curved or non planar cracks $[14,15,16]$. Some domain integral formulations rely on extraction fields based on the first term of the asymptotic solution for a crack in LEFM. It is important to note that those fields correspond to a straight crack. As an example, the interaction integral uses explicitly auxiliary fields in its formulation. The solution fields for curved cracks are not the same as the fields for the straight crack and a generic expression for this case is not available. The present convergence study also analyzes the influence of different extraction $q$-functions for curved crack problems.

The use of the straight-crack auxiliary fields introduces some modifications in the interaction integral when applied to curved cracks that can be affected by the type of the virtual velocity function $q_{i}$. In this work, the influence of different definitions of the virtual velocity function $q_{i}$ is also analyzed. However if a small domain extraction is used, the effect of using the straight crack fields, instead of using the correct curved crack fields, can be minimized [14]. The orthogonality of the level set basis is also better fulfilled if only a small domain is considered. The disadvantage is that a high level of refinement is needed when using a small extraction domain.

In summary, several factors can affect the performance of domain integrals when applied to the analysis of curved cracks using XFEM and level sets. In this work, the influence of these factors is studied, namely:

- The influence of the basis orthogonality. The domain integral formulation relies on relationships that accept basis orthogonality to avoid the contour integration along the crack faces. However, the basis orthogonality cannot be guaranteed in curved crack problems using level sets.

- The auxiliary fields used in the interaction integral correspond to the straight crack case. However, in the literature their application is usually extended to the analysis of curved cracks. Explicitly, the strain auxiliary field is derived from other auxiliary fields (either displacement or stress fields) using relationships that do not hold for curved cracks. An analysis of the effect of the auxiliary strain field definition is performed in this work to assess the behaviour of different choices for the auxiliary strain field. 
- In addition, the validity of the reciprocity condition in some of the terms of the interaction integral when using the straight crack auxiliary fields in curved configurations is also discussed.

- The path independence of the interaction integral is affected by the former issues. Consequently, the influence of the virtual velocity function $q_{i}$ and the extraction domain size in curved crack problems is also analyzed.

\section{XFEM}

The XFEM is a numerical method that enables a local enrichment of the FE approximation spaces. The enrichment is realized through the partition of unity concept. The method is useful for the approximation of solutions with pronounced non-smooth characteristics in small parts of the computational domain, for example near discontinuities and singularities. In these cases, standard numerical methods such as the FEM often exhibit poor accuracy. The essential idea in XFEM is to use a displacement field approximation that can model any arbitrary discontinuity as the neartip asymptotic crack field. As a consequence it is not necessary to modify the mesh to consider a specific crack; at most, moderate refinement must be introduced around the crack to achieve a good accuracy in elastic fracture mechanics. The method is based on the enrichment of the FE model with additional degrees of freedom (DOFs) that are tied to the nodes of the elements affected by the crack [1].

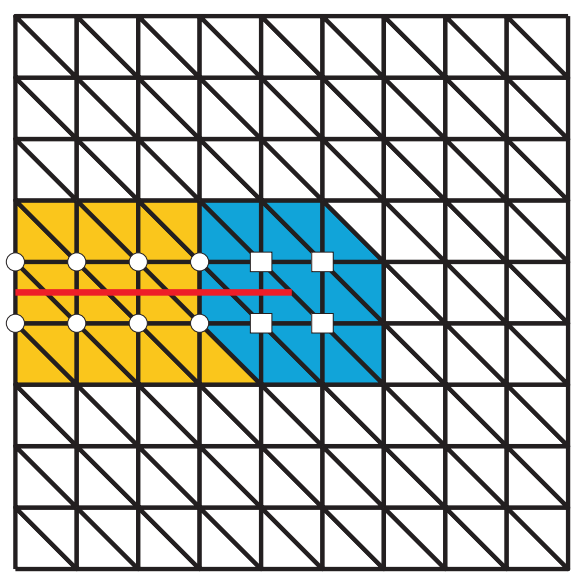

Figure 1. Enriched nodes in the X-FEM. Circles: nodes with Heaviside function, two additional DOFs. Elements affected by this enrichment in yellow. Squares: nodes with crack tip enrichment function, eight additional DOFs. Elements affected by this enrichment in blue.

Elements that contain at least one enriched node are known as enriched elements (see Fig. 1). Nodes with two additional DOFs (one for each coordinate direction) have shape functions that multiply the Heaviside function $H(\mathbf{x})$ (function of unit magnitude whose sign changes across the crack, $H(\mathbf{x})= \pm 1$ ). This function introduces the discontinuity across the crack faces. Nodes with 
eight additional DOFs are enriched in the two cartesian directions with four crack tip functions $F_{\alpha}(\mathbf{x})[1]$ :

$$
\left[F_{\alpha}(r, \theta), \alpha=1-4\right]=\left[\sqrt{r} \sin \frac{\theta}{2}, \sqrt{r} \cos \frac{\theta}{2}, \sqrt{r} \sin \frac{\theta}{2} \sin \theta, \sqrt{r} \cos \frac{\theta}{2} \sin \theta\right]
$$

where $r, \theta$ are the local polar coordinates defined at the crack tip. We note that the span of the above functions can reproduce the asymptotic displacement fields in LEFM, which gives rise to the neartip singular behavior in strains and stresses. It is well-documented in the literature [1, 6], and also verified through our studies that these functions significantly improve the accuracy of the different SIF extraction.

The displacement approximation for crack modeling in the extended finite element method takes the form [1]:

$$
\mathbf{u}_{\mathrm{xfem}}(\mathbf{x})=\sum_{i \in \mathcal{I}} N_{i}(\mathbf{x}) \mathbf{u}_{i}+\sum_{i \in \mathcal{J}} N_{i}(\mathbf{x}) H(\mathbf{x}) \mathbf{a}_{i}+\sum_{i \in \mathcal{K}}\left[N_{i}(\mathbf{x}) \sum_{\alpha=1}^{4} F_{\alpha}(\mathbf{x}) \mathbf{b}_{i \alpha}\right]
$$

where $\mathcal{I}$ is the set of all nodes in the mesh, $N_{i}(\mathbf{x})$ is the nodal shape function and $\mathbf{u}_{i}$ is the standard DOF of node $i$ ( $\mathbf{u}_{i}$ represents the physical nodal displacement for non-enriched nodes only). The subsets $\mathcal{J}$ and $\mathcal{K}$ contain the nodes enriched with Heaviside function $H(\mathbf{x})$ or crack-tip functions $F_{\alpha}(\mathbf{x})$, respectively, and $\mathbf{a}_{i}, \mathbf{b}_{i \alpha}$ are the corresponding DOFs.

As in the standard FEM, it is necessary to perform numerical integration over the element domain to compute the element stiffness matrix. However, the elements that contain the crack include a displacement discontinuity due to the XFEM formulation. These elements are subdivided into sub-domains in which the crack is one of the sub-domain boundaries to carry out the numerical integrations. The algorithm presented in [18] is used to subdivide the elements totally cut by the crack. The integration on the normal elements or on the sub-domains corresponding to the cut elements is performed using a normal Gauss-Legendre integration rule. The element affected by the crack tip is subdivided and integrated using a quasi polar rule introduced in [19]. An example of a generic subdivision can be observed in Fig. 2.

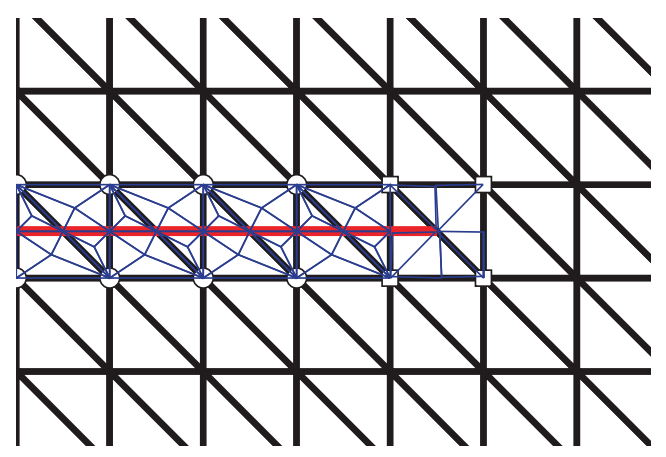

Figure 2. Example of subdivision of elements intersected by a crack for integration purposes. 


\section{LEVEL SETS}

In addition to the numerical method, a description of the crack geometry is needed. The information of the crack is introduced through the use of distance functions based on level sets [3, 4, 11]. Two level sets are used to perform the crack description. They are denoted as $\phi$ and $\psi$ and can be observed in the figure 3. The $\phi$ level set is called the crack surface level set and its zero value corresponds to the crack surface. The $\psi$ level set, called front level set, determines the position relative to the crack tip, which is located at the intersection of $\phi=0$ with $\psi=0$, and it is desirable that be orthogonal to the level set $\phi$. In summary, the crack is given by

$$
\begin{array}{ll}
\phi(\mathbf{x})=0 & \psi(\mathbf{x})<0 \longrightarrow \text { defines crack location } \\
\phi(\mathbf{x})=0 & \psi(\mathbf{x})=0 \longrightarrow \text { defines crack tip location }
\end{array}
$$

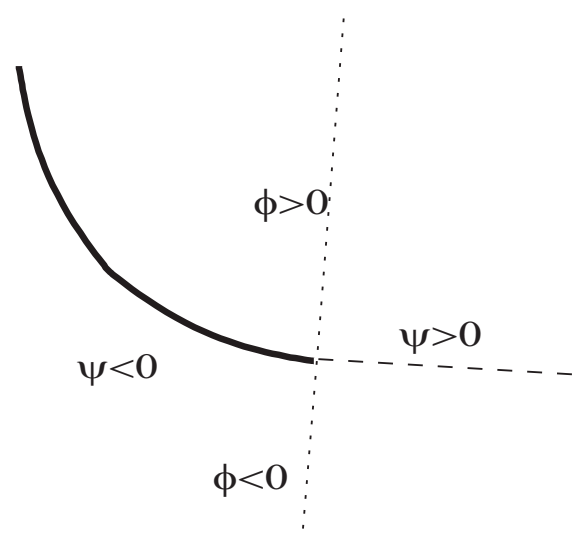

Figure 3. Example of level sets for the general description of a crack.

The use of level sets for describing the crack has other advantages. First, the evaluation of the level set at the nodes of the elements allows to select the elements to be enriched. It is achieved just observing the change of sign of the distance functions in the element. Nodes that need to be enriched are the nodes which fulfill the conditions 4 where $I^{e l}$ is the set of nodes belonging to a given element $[3,11]$ :

$$
\begin{aligned}
& \left\{\begin{array}{l}
\min _{i \in I^{e l}}\left(\operatorname{sign}\left(\phi_{i}(x, y)\right)\right) \max _{i \in I^{e l}}\left(\operatorname{sign}\left(\phi_{i}(x, y)\right)\right) \leq 0 \\
\max _{i \in I^{e l}}\left(\operatorname{sign}\left(\psi_{i}(x, y)\right)\right)<0
\end{array} \longrightarrow\right. \text { Heaviside enrichment } \\
& \left\{\begin{array}{l}
\min _{i \in I^{e l}}\left(\operatorname{sign}\left(\phi_{i}(x, y)\right)\right) \max _{i \in I^{e l}}\left(\operatorname{sign}\left(\phi_{i}(x, y)\right)\right) \leq 0 \\
\min _{i \in I^{e l}}\left(\operatorname{sign}\left(\psi_{i}(x, y)\right)\right) \max _{i \in I^{e l}}\left(\operatorname{sign}\left(\psi_{i}(x, y)\right)\right) \leq 0
\end{array} \longrightarrow\right. \text { Crack tip enrichment }
\end{aligned}
$$

Moreover the level set distance functions $\phi$ and $\psi$ can be used to build a curvilinear local basis associated with the crack geometry. The general local basis is defined using the normalized gradients of the level set functions 5 as in [3]. The level set local basis defines a natural system of coordinates 
that represents the crack magnitudes. All tensors used are represented in this coordinate system and the derivatives for curved cracks are built following $[15,16]$.

$$
\begin{aligned}
& \mathbf{e}_{1}=\frac{\nabla \psi}{\|\nabla \psi\|} \\
& \mathbf{e}_{2}=\frac{\nabla \phi}{\|\nabla \phi\|}
\end{aligned}
$$

Usually, it is assumed that the level sets are orthogonal in the sense that $\nabla \phi \nabla \psi=0$, but this is not true for the general case. However we can ensure that the basis vectors are orthogonal if a vector is built using one level set and the other is made orthogonal to the former, as given in 6 following [11].

$$
\begin{aligned}
& \mathbf{e}_{1}=\frac{\nabla \psi}{\|\nabla \psi\|} \\
& \mathbf{e}_{2} \perp \mathbf{e}_{1}
\end{aligned}
$$

In our implementation the values of the level sets are stored at the nodes of the finite element mesh. The customary element shape functions are used for interpolating within the domain. The values of the vectors of the local basis, in the description of curved cracks as straight segments within each element, are computed at each element and averaged at the shared nodes with the neighboring elements $[4,11]$. To some extent, this maintains the curvature and allows to obtain an approximation to a continuous smooth local basis variation.

Another advantage that arises from the use of level sets and its associated local basis is the possibility of building polar coordinates at the crack tip, which provide directly $r$ and $\theta$ for the crack tip enrichment functions 1 as given in 7. The Heaviside enrichment matches the sign of the $\phi$ level set function. Fig. 4 shows an example for the problem analyzed in this work.

$$
\begin{aligned}
& r=\sqrt{\psi^{2}+\phi^{2}} \\
& \theta=\tan ^{-1} \frac{\phi}{\psi}
\end{aligned}
$$

Therefore, use of level sets is necessary, because it enables the computation of the $(r, \theta)$ coordinates for curved cracks, which in turn are necessary for computing the SIFs. Note that for points located ahead the crack tip it would be easy to compute $(r, \theta)$ using a local cartesian reference system (with origin at the crack tip), but it would not be correct for points behind the crack tip (for example, points on the curved crack faces). Hence, a curvilinear reference system becomes necessary and the level sets provide an appropriate system of coordinates. 


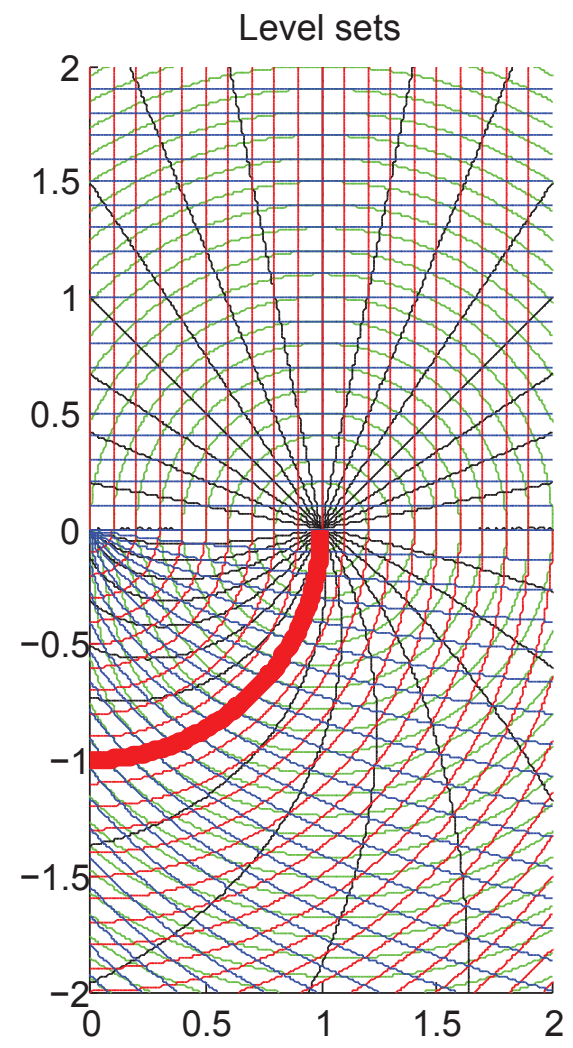

Figure 4. Level sets for an arc crack. Red and blue lines are $\phi$ and $\psi$ levels. Green lines represent $r$ and black lines the angle $\theta$ as defined in 7 . The exact crack geometry corresponds to the thick red line.

\section{J-INTEGRAL}

The $J$-integral was introduced in [7] and it is one of the most powerful tools available for the extraction of the SIF, especially in its domain form [8]. However it does not allow the extraction of the SIF in mixed mode situations. The domain form is given by:

$$
J=-\int_{A} P_{i j} \partial_{j} q_{i} \mathrm{~d} \Omega
$$

where $P_{i j}$ is the Eshelby's tensor [20], which is defined as

$$
P_{i j}=\frac{1}{2} \varepsilon_{k l} \sigma_{k l} \delta_{i j}-\sigma_{j k} \partial_{i} u_{k}
$$

where $u_{i}$ is the displacement field, $\sigma_{i j}$ is the stress field, $\delta_{i j}$ is the Kronecker's delta function and $q_{i}$ is a vector in direction $\mathbf{e}_{1}$ with a modulus proportional to a weight function $\alpha(\mathbf{x})$, which is zero on the contour of the extraction domain $A$ and one at the crack tip point. For 2D problems the SIFs can be related to the $J$-integral using the expression

$$
J=\frac{K_{\mathrm{I}}^{2}}{E^{*}}+\frac{K_{\mathrm{II}}^{2}}{E^{*}}
$$


where $E^{*}$ is the modified Young's modulus defined in terms of material parameters $E$ (Young's modulus) and $\nu$ (Poisson's ratio) as

$$
E^{*}= \begin{cases}E & \text { plane stress } \\ \frac{E}{1-\nu^{2}} & \text { plane strain }\end{cases}
$$

\section{INTERACTION INTEGRAL}

The interaction integral is used to extract the SIF under mixed-mode situations, $[3,9,10,11]$, enabling the computation of $K_{\mathrm{I}}$ and $K_{\mathrm{II}}$ in $2 \mathrm{D}$ problems. To achieve this goal, auxiliary fields are needed. The interaction integral is derived from the application of the $J$-integral to a problem where two stress fields are involved, resulting in the following decomposition:

$$
J^{(1+2)}=J^{(1)}+J^{(2)}+I
$$

The term $I$ corresponds to the interaction integral and includes the interaction between the two intervening fields. For straight cracks the interaction integral is:

$$
I=-\int_{A}\left(\sigma_{k l} \varepsilon_{k l}^{a u x} \delta_{i j}-\sigma_{k j}^{a u x} \partial_{i} u_{k}-\sigma_{k j} \partial_{i} u_{k}^{a u x}\right) \partial_{j} q_{i} \mathrm{~d} \Omega
$$

The fields denoted with the superscript ${ }^{\text {aux }}$ are the auxiliary fields. Usually, the auxiliary fields are selected to be the straight crack fields, allowing the extraction of the different SIF modes. The fields $u_{i}, \sigma_{i j}, q_{i}$ and $\delta_{i j}$ are defined in the same way as in the $J$-integral, being $\varepsilon_{i j}$ the strain field. The use of the straight crack fields as auxiliary fields to evaluate a curved crack configuration, implies that 13 is not longer valid. The usual expression used to evaluate the domain form of this integral for curved cracks $[1,3,11]$ is:

$$
\begin{aligned}
I= & -\int_{A}\left(\sigma_{k l} \varepsilon_{k l}^{a u x} \delta_{i j}-\sigma_{k j}^{a u x} \partial_{i} u_{k}-\sigma_{k j} \partial_{i} u_{k}^{a u x}\right) \partial_{j} q_{i} \mathrm{~d} \Omega \\
& -\int_{A}\left(\partial_{i} \sigma_{k l}^{a u x} \varepsilon_{k l} \delta_{i j}-\sigma_{k l} \partial_{l i} u_{k}^{a u x}-\partial_{l} \sigma_{k l}^{a u x} \partial_{i} u_{k}\right) q_{i} \mathrm{~d} \Omega
\end{aligned}
$$

The derivation of the expression 14 can be found in [10]. The generic treatment to apply the interaction integral to curved cracks is presented in $[15,16]$ and it is also analyzed for the $J$-integral in [17]. It is important to note that all the hypothesis assumed when deriving the above interaction integral expression, using the straight crack fields as auxiliary fields, should not be accepted directly for generic curved cracks $[10,14,15,16]$. However, they are all admitted in $[15,16]$.

We will justify a proposal of modification of the interaction integral for curved cracks recalling its derivation. With no simplifications the general form of the interaction integral is

$$
\begin{aligned}
I= & -\int_{A}\left(\frac{1}{2}\left(\varepsilon_{k l}^{a u x} \sigma_{k l}+\varepsilon_{k l} \sigma_{k l}^{a u x}\right) \delta_{i j}-\sigma_{k j}^{a u x} \partial_{i} u_{k}-\sigma_{k j} \partial_{i} u_{k}^{a u x}\right) \partial_{j} q_{i} \mathrm{~d} \Omega \\
& -\int_{A}\left(\frac{1}{2}\left(\partial_{j} \varepsilon_{k l}^{a u x} \sigma_{k l}+\varepsilon_{k l}^{a u x} \partial_{j} \sigma_{k l}+\partial_{j} \varepsilon_{k l} \sigma_{k l}^{a u x}+\varepsilon_{k l} \partial_{j} \sigma_{k l}^{a u x}\right) \delta_{i j}\right) q_{i} \mathrm{~d} \Omega \\
& -\int_{A}\left(-\partial_{j} \sigma_{k j}^{a u x} \partial_{i} u_{k}-\sigma_{k j}^{a u x} \partial_{i j} u_{k}-\partial_{j} \sigma_{k j} \partial_{i} u_{k}^{a u x}-\sigma_{k j} \partial_{i j} u_{k}^{a u x}\right) q_{i} \mathrm{~d} \Omega
\end{aligned}
$$


The first problem arises from the fact that the inner equilibrium equation and the compatibility equations between strains-displacements of the auxiliary fields do not hold for a curved crack studied in curvilinear coordinates, that is

$$
\begin{aligned}
& \partial_{i} \sigma_{i j}^{a u x} \neq 0 \\
& \left(\partial_{l i} u_{j}^{a u x}-\partial_{l} \varepsilon_{i j}^{a u x}\right) \neq 0
\end{aligned}
$$

Therefore, the expression 13 cannot be used in curved cracks. In addition, the reciprocity relationship is also assumed in the derivation of 14 . The reciprocity condition implies that the same constitutive tensor is used to relate the auxiliary strain field and the auxiliary stress field, i.e.

$$
\varepsilon_{i j}^{a u x} \sigma_{i j}=\varepsilon_{i j}^{a u x} C_{i j k l} \varepsilon_{k l}=C_{k l i j} \varepsilon_{i j}^{a u x} \varepsilon_{k l}=\sigma_{k l}^{a u x} \varepsilon_{k l}
$$

If this reciprocity condition is assumed, then the following relationship holds:

$$
\frac{1}{2}\left(\varepsilon_{i j}^{a u x} \sigma_{i j}+\varepsilon_{i j} \sigma_{i j}^{a u x}\right)=\varepsilon_{i j}^{a u x} \sigma_{i j}
$$

As a consequence, Eq. 14 is obtained as a simplification of Eq. 15, as is usually done in the literature. The terms involved in 18 correspond to the interaction strain energy. This way, the auxiliary fields corresponding to a straight crack are enforced to adjust to a curved coordinate system. This is the reason why the reciprocity relationship cannot be accepted and the simplifications 18 and 14 should not be used for curved crack problems.

The reciprocity relationship is also used in the derivation of the second integrand of 14 from Eq. 15, i.e.

$$
\partial_{i} \sigma_{k l}^{a u x} \varepsilon_{k l} \delta_{i j}-\sigma_{k l} \partial_{l i} u_{k}^{a u x}-\partial_{l} \sigma_{k l}^{a u x} \partial_{i} u_{k}
$$

is a simplification of

$$
\begin{aligned}
& \frac{1}{2}\left(\partial_{j} \varepsilon_{k l}^{a u x} \sigma_{k l}+\varepsilon_{k l}^{a u x} \partial_{j} \sigma_{k l}+\partial_{j} \varepsilon_{k l} \sigma_{k l}^{a u x}+\varepsilon_{k l} \partial_{j} \sigma_{k l}^{a u x}\right) \delta_{i j} \\
& -\partial_{j} \sigma_{k j}^{a u x} \partial_{i} u_{k}-\sigma_{k j}^{a u x} \partial_{i j} u_{k}-\partial_{j} \sigma_{k j} \partial_{i} u_{k}^{a u x}-\sigma_{k j} \partial_{i j} u_{k}^{a u x}
\end{aligned}
$$

If the reciprocity relationship is not applied in 20, the computation of derivatives of the numerical approximation near the crack face are required, such as $\partial_{j} \sigma_{k l}$ and $\partial_{j} \varepsilon_{k l}$. The numerical computation of these derivatives introduce large numerical errors. However, we have verified that the integrand 20 has little influence on the final result. Hence, to avoid the introduction of further numerical errors, we will admit the reciprocity relationship only in the derivation of the simplified expression 19. Therefore, the interaction integral expression that we will use in this work is finally:

$$
\begin{aligned}
I= & -\int_{A}\left(\frac{1}{2}\left(\sigma_{k l} \varepsilon_{k l}^{a u x}+\sigma_{k l}^{a u x} \varepsilon_{k l}\right) \delta_{i j}-\sigma_{k j}^{a u x} \partial_{i} u_{k}-\sigma_{k j} \partial_{i} u_{k}^{a u x}\right) \partial_{j} q_{i} \mathrm{~d} \Omega \\
& -\int_{A}\left(\partial_{i} \sigma_{k l}^{a u x} \varepsilon_{k l} \delta_{i j}-\sigma_{k l} \partial_{l i} u_{k}^{a u x}-\partial_{l} \sigma_{k l}^{a u x} \partial_{i} u_{k}\right) q_{i} \mathrm{~d} \Omega
\end{aligned}
$$




\subsection{Auxiliary fields}

Due to the lack of knowledge of a general expression for the auxiliary fields for curved cracks, the first terms of the Williams' asymptotic fields of the LEFM are used as auxiliary fields, using the definitions stated in 7 as $\theta$ and $r$. However, it is important to note that for general curved cracks, the conditions of compatibility, inner equilibrium and Hooke's law are not simultaneously verified for this selection of the auxiliary fields $[14,15,16]$. The auxiliary displacement field is the first term of the Williams' series expansion:

$$
\begin{aligned}
& u_{1}^{a u x}=\frac{1}{2 \mu} \sqrt{\frac{r}{2 \pi}}\left\{K_{\mathrm{I}}^{\text {aux }}(\kappa-\cos \theta) \cos \frac{\theta}{2}+K_{\mathrm{II}}^{\text {aux }}(\kappa+2+\cos \theta) \sin \frac{\theta}{2}\right\} \\
& u_{2}^{a u x}=\frac{1}{2 \mu} \sqrt{\frac{r}{2 \pi}}\left\{K_{\mathrm{I}}^{\text {aux }}(\kappa-\sin \theta) \sin \frac{\theta}{2}+K_{\mathrm{II}}^{\text {aux }}(\kappa-2+\cos \theta) \cos \frac{\theta}{2}\right\}
\end{aligned}
$$

where

$$
\mu=\frac{E}{2(1+\nu)}, \quad \kappa= \begin{cases}3-4 \nu & \text { plane strain } \\ \frac{3-\nu}{1+\nu} & \text { plane stress }\end{cases}
$$

The auxiliary stress field is:

$$
\begin{aligned}
\sigma_{11}^{\text {aux }} & =\frac{K_{\mathrm{I}}^{\text {aux }}}{\sqrt{2 \pi r}}\left[1-\sin \frac{\theta}{2} \sin \frac{3 \theta}{2}\right] \cos \frac{\theta}{2}-\frac{K_{\mathrm{II}}^{\text {aux }}}{\sqrt{2 \pi r}}\left[2+\cos \frac{\theta}{2} \cos \frac{3 \theta}{2}\right] \sin \frac{\theta}{2} \\
\sigma_{22}^{\text {aux }} & =\frac{K_{\mathrm{I}}^{\text {aux }}}{\sqrt{2 \pi r}}\left[1+\sin \frac{\theta}{2} \sin \frac{3 \theta}{2}\right] \cos \frac{\theta}{2}+\frac{K_{\mathrm{II}}^{\text {aux }}}{\sqrt{2 \pi r}} \cos \frac{\theta}{2} \cos \frac{3 \theta}{2} \sin \frac{\theta}{2} \\
\sigma_{12}^{\text {aux }} & =\frac{K_{\mathrm{I}}^{\text {aux }}}{\sqrt{2 \pi r}} \cos \frac{\theta}{2} \cos \frac{3 \theta}{2} \sin \frac{\theta}{2}+\frac{K_{\mathrm{II}}^{\text {aux }}}{\sqrt{2 \pi r}}\left[1-\sin \frac{\theta}{2} \sin \frac{3 \theta}{2}\right] \cos \frac{\theta}{2}
\end{aligned}
$$

The auxiliary field for the strains is also needed. Two possible choices arise to obtain the strain field. The first choice is based on the enforcement of the constitutive strain-stress relationship [3, 11] and the strain field is computed from the stress field by applying the Hooke's law.

$$
\varepsilon_{i j}^{a u x}=C_{i j k l}^{-1} \sigma_{k l}^{a u x}
$$

The second option is that the strain field can be obtained from the derivatives of the displacement field like in $[10,15,16]$, enforcing the strain-displacement relationship:

$$
\varepsilon_{i j}^{a u x}=\left\{\nabla_{s i m} \mathbf{u}^{a u x}\right\}_{i j}
$$

In principle, there is no reason to assume that either choice is the best one. In this paper, we propose a third option. As neither expression verifies simultaneously the compatibility and inner equilibrium relationships for the auxiliary fields, an averaged strain field between the two options can also be considered. The objective is to verify an averaged condition between compatibility and inner equilibrium relationship. The averaged auxiliary strain field is given by

$$
\varepsilon_{i j}^{a u x}=\frac{\left\{\nabla_{\operatorname{sim}} \mathbf{u}^{a u x}\right\}_{i j}+C_{i j k l}^{-1} \sigma_{k l}^{a u x}}{2}
$$


The gradients of the auxilary fields in curvilinear coordinates need the inclusion of additional terms, that involve the Christoffel symbols, related with curvature effects. The detailed implementation of this derivatives can be found in $[15,16]$.

\subsection{SIF extraction}

The well-known relationship between the interaction integral and the SIFs is derived from the expressions 10 and 12:

$$
I=\frac{2}{E^{*}}\left(K_{\mathrm{I}} K_{\mathrm{I}}^{a u x}+K_{\mathrm{II}} K_{\mathrm{II}}^{a u x}\right)
$$

If the SIFs of the auxiliary field are chosen as $K_{\mathrm{I}}^{a u x}=1$ and $K_{\mathrm{II}}^{\text {aux }}=0$, then the sought value $K_{\mathrm{I}}$ is obtained as:

$$
I=\frac{2}{E^{*}} K_{\mathrm{I}}
$$

Similarly, if $K_{\mathrm{I}}^{a u x}=0$ and $K_{\mathrm{II}}^{a u x}=1$ then

$$
I=\frac{2}{E^{*}} K_{\mathrm{II}}
$$

\section{DOMAIN INTEGRAL COMPUTATION IN XFEM}

The integrals 8, 14, 13 and 15 are expressed in domain formulation which uses the virtual velocity field $q_{i}[3,11]$. The equivalent contour formulation is not well suited for numerical computations in the finite element framework. As a consequence, the selection of the virtual velocity field needs to be addressed. The extraction domain is controlled through the use of the virtual velocity field and the numerical integration is performed within the extraction domain using the integration points at the element level. The virtual velocity field must be tangent to the crack face and is defined by the expression:

$$
\mathbf{q}=\alpha(\phi, \psi) \mathbf{e}_{1}
$$

Note that the domain extraction region and the weight of the integration on the extraction domain is controlled through the use of $\alpha$, which depends on the level set coordinates. The function $\alpha$ has to take unit value at the crack tip and zero on the boundary of the extraction domain.

For the sake of simplicity the extraction domain has been selected with a boundary defined by a circle of radius $R$ which will depend on the crack curvature radius $R_{c}$. The selection of the exact functional definition for $\alpha$ needs here some discussion. The $\alpha$ function is evaluated using a finite element approach, i.e. it is evaluated at nodes and interpolated within the element domain using the finite element shape functions. We have considered two possibilities for the definition of $\alpha$ at nodes: a plateau function and a ramp function.

One of the most used $\alpha$ functions is the plateau function. It has the advantage that in the central region $\alpha$ is constant and its derivative vanishes (and so does the derivative of $\mathbf{q}$ ). This is not strictly true for curved cracks as the gradient involves the use of curvilinear coordinates and Christoffel 
symbols, leading to nonzero results that depend on the curvature. In any case, we have verified this is a small quantity. In the central region, the fields corresponding to the singular term dominate and even with the introduction of the XFEM, enrichment of this central part introduces numerical errors that suggest to reduce it to a minimum. The exact plateau considered is:

$$
\alpha= \begin{cases}1 & \text { if } \sqrt{\phi^{2}+\psi^{2}}<R \\ 0 & \text { if } \sqrt{\phi^{2}+\psi^{2}} \geq R\end{cases}
$$

Note that with our implementation Eq. 31 corresponds to an integration over a one-element width ring. The interaction integral for curved cracks 15 includes a term which involves $q_{i}$. For straight cracks this term is zero as all the assumptions for the auxiliary fields are fulfilled. For curved crack problems this term will dominate in the central region if a plateau function is selected. Another choice for the $\alpha$ function is the ramp function 32. However, the ramp function has the drawback of using information of points close to the crack tip. As the $J$-integral has no terms involving explicitly $q_{i}$ (only its spatial derivative), the use of the ramp function in combination with the $J$-integral has not a large influence as compared to the interaction integral.

$$
\alpha= \begin{cases}1-\frac{\sqrt{\phi^{2}+\psi^{2}}}{R} & \text { if } \sqrt{\phi^{2}+\psi^{2}}<R \\ 0 & \text { if } \sqrt{\phi^{2}+\psi^{2}} \geq R\end{cases}
$$

\section{REFERENCE PROBLEM}

The problem considered is an arc crack in an infinite plate subjected to equibiaxial stress. The geometry of the crack is defined by the radius $R_{c}$ and the angle $\beta$, as shown in Figure 5. In this study we have taken the values $R_{c}=1$ and two $\beta$ angles, $\beta=\pi / 2$ for the main study and $\beta=\pi / 4$ as a further verification. The domain used for the XFEM analyses is a finite portion centered at the crack tip and defined by a width $w=2 a$ and a height $h=4 a$, where $a=R_{c} \sin (\beta)$ is related to the crack length. The analytical stress field solution and symmetry conditions are imposed on the boundary of the finite domain in order to make the model equivalent to the infinite domain problem. The analytical solution to this problem is given in [21]. This problem is well studied in the bibliography, e.g. [16, 22]. The following convergence rate study for this reference problem is carried out using a mesh sequence with regular linear triangular elements with uniform refinement. The reference solution for the SIFs is:

$$
\begin{aligned}
& K_{\mathrm{I}}^{e x}=\sigma_{\infty}(\pi a)^{\frac{1}{2}} \frac{\cos \left(\frac{\beta}{2}\right)}{1+\sin ^{2}\left(\frac{\beta}{2}\right)} \\
& K_{\mathrm{II}}^{e x}=\sigma_{\infty}(\pi a)^{\frac{1}{2}} \frac{\sin \left(\frac{\beta}{2}\right)}{1+\sin ^{2}\left(\frac{\beta}{2}\right)}
\end{aligned}
$$

where $\sigma_{\infty}$ is the applied remote load.

The mesh sequence, used for the convergence study, is built using regular triangular elements with a side length defined by the following series: 


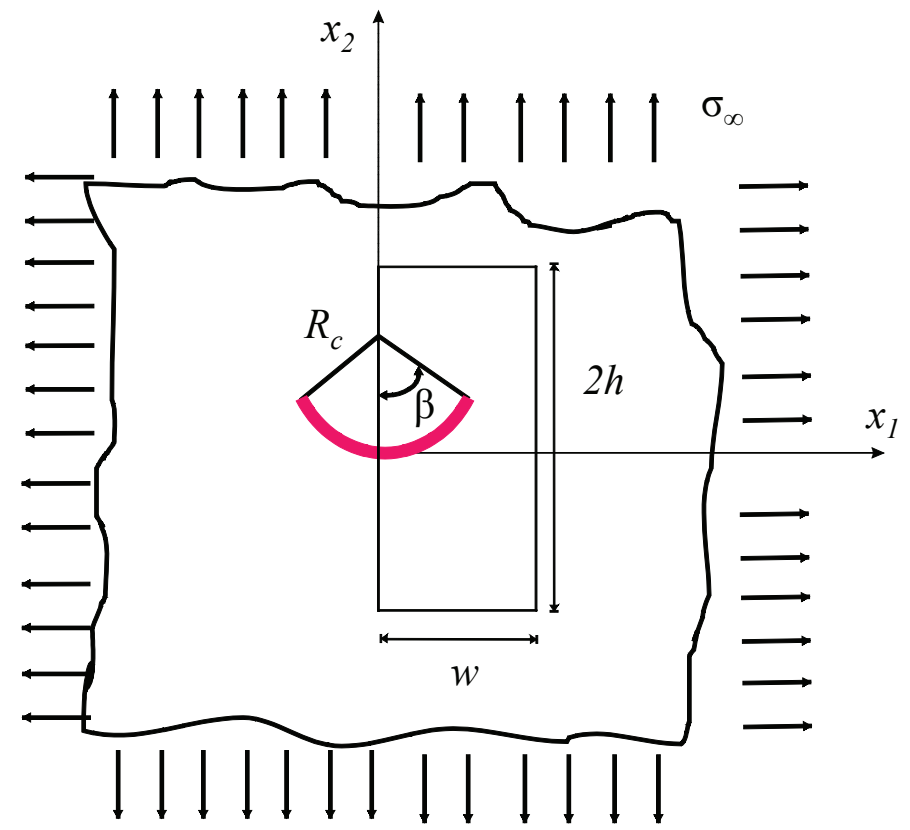

Figure 5. The problem of an arc crack in an infinitely extended plate subjected to equibiaxial tension

$$
\begin{aligned}
h= & \left\{\frac{2 a}{63}, \frac{2 a}{73}, \frac{2 a}{93}, \frac{2 a}{113}, \frac{2 a}{133}, \frac{2 a}{163}, \frac{2 a}{173}, \frac{2 a}{193}, \frac{2 a}{213},\right. \\
& \left.\frac{2 a}{233}, \frac{2 a}{257}, \frac{2 a}{323}, \frac{2 a}{413}, \frac{2 a}{457}, \frac{2 a}{513}, \frac{2 a}{673}\right\}
\end{aligned}
$$

The first mesh of the sequence can be observed in Figure 6. In our model the curved crack is described as straight segments within each element. This introduces further approximations, although the associated error is deemed small for sufficiently refined meshes, as the ones used in this analysis. It is possible to improve the crack description using higher order elements [23], but this issue has not been considered in this work. 


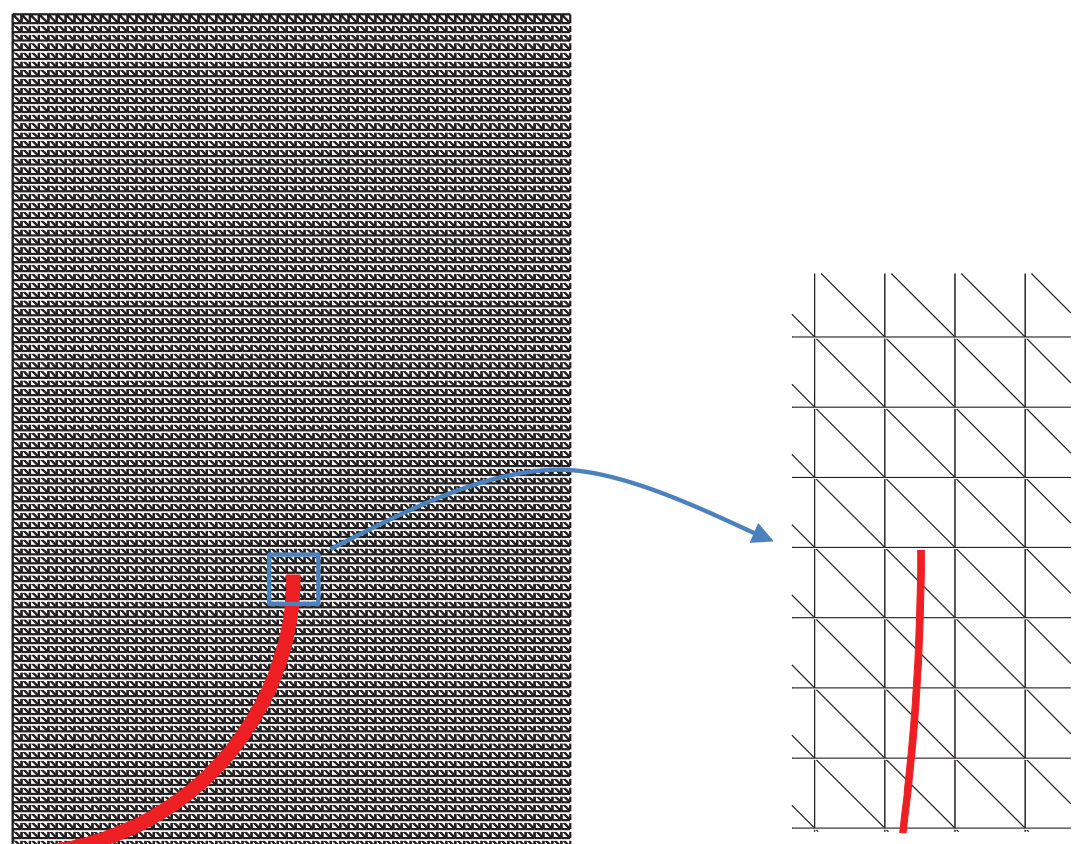

Figure 6. First mesh of the sequence for the arc-crack problem and enlarged view of the vicinity of the crack tip. $\beta=\pi / 2$.

\section{ANALYSIS OF CONVERGENCE RATES}

The convergence is studied computing the error in the SIF versus the number of degrees of freedom of the mesh. The expected optimal convergence rate for the error in the SIF using XFEM with topological enrichment is $0.5[19,24,25,26,27]$.The first study presented here is the effect of the selection of the level set basis. The extraction domain radius is selected to be $R=0.2 R_{c}$. The $\alpha$ ramp function 32 is used. It is worth noting that we are using an extraction domain and $\alpha$ function that magnify the errors derived from the basis definition. Two choices of the level set basis are studied: on the one hand, the basis is formulated directly from the level set definition 5. On the other hand, an orthogonal basis is defined using only one level set and geometric considerations that ensure orthogonality 6. According to Figure 7, the latter choice (the orthogonal basis) yields the correct convergence rate whereas the basis built directly from the level sets does not converge with the optimum rate. The requirement of orthogonality has also been proposed and recommended by other authors, e.g. Duflot [11], although a comparative convergence study is not presented in [11].

Once the correct choice for the basis has been evidenced, we move now to the analysis of the interaction integral. The same extraction domain is used, i.e. $\alpha$ is defined by a ramp function 


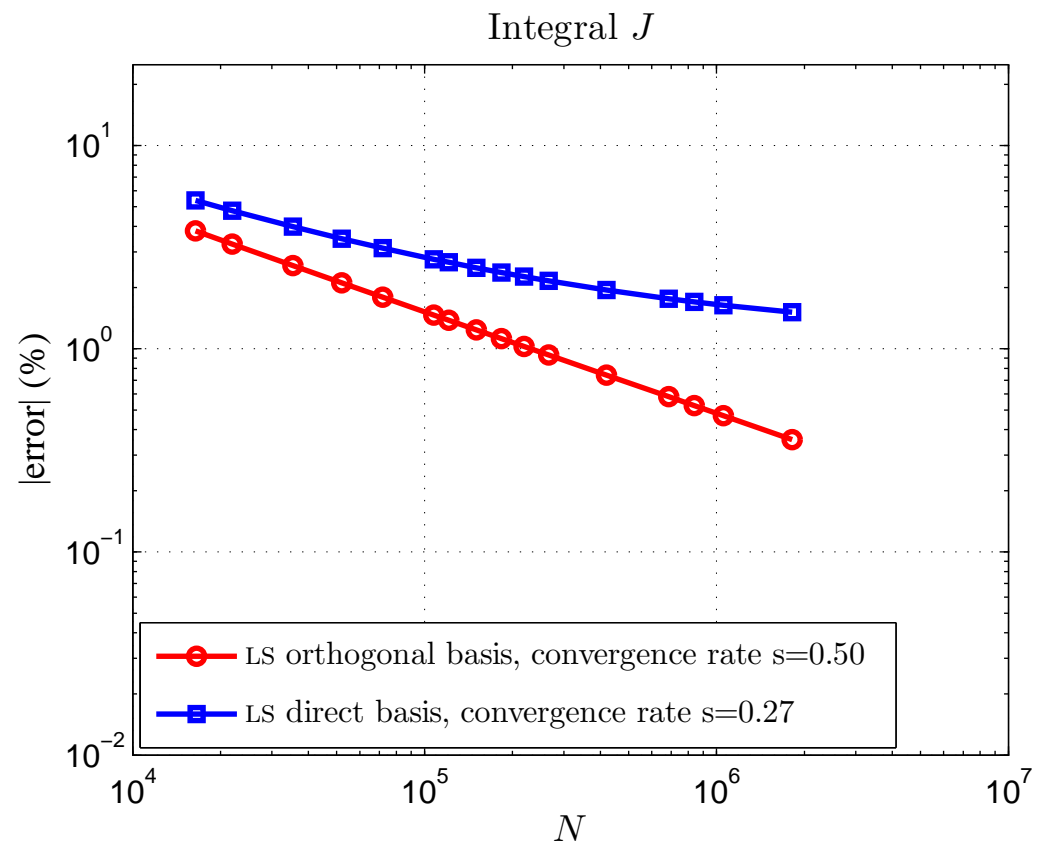

Figure 7. Level sets local basis definition study. Error convergence study on Integral results.

with $R=0.2 R_{c}$. Five studies have been carried out considering the different options explained in previous sections. The different options are: a) auxiliary strain field obtained from auxiliary displacements 25 and usual interaction integral expression 14; b) auxiliary strain field obtained from the auxiliary stress field 24 and the usual interaction integral expression; c) auxiliary strain field defined using the averaged field 26 and the usual interaction integral expression; d) auxiliary strain field obtained from auxiliary displacement field with the modified interaction integral formulation 21, denoted in the plots as energy term; e) auxiliary averaged strain field with modified interaction integral, also denoted in the plots as energy term. The effect on the convergence rate for the error in $K_{\mathrm{I}}$ and $K_{\mathrm{II}}$ can be observed in Figures 8 and 9.

The optimum convergence rate is 0.5 but none of our studies achieves that rate. It is observed that the case $\varepsilon^{a u x}$ obtained from $\sigma^{a u x}$ is more accurate for $K_{\mathrm{I}}$ than the other cases, but a sign change is detected in the approach error shown by the abrupt change in slope (the plotted magnitude is the absolute value of the error). This sign change is indicative of convergence to a different solution. No significant differences can be observed in the behavior for the error in $K_{\mathrm{II}}$. From Figure 8 it can be inferred that the best behavior can be associated with the use of the averaged auxiliary strain field and the modified interaction integral expression, since it shows the best convergence rate and accuracy converging to the exact solution. We remark that this choice is the one that introduces less assumptions, since the reciprocity relationship is only assumed for the second term in Eq. 21 and it uses an average approximation for the strain auxiliary field to fulfill the inner equilibrium and compatibility conditions.

In what follows, the effect of the extraction function $\alpha$ is analyzed: the plateau function defined in 31 and the ramp function 32. This part of the study is performed with both integrals, the interaction integral and the $J$-integral. The same domain extraction radius is used, $R=0.2 R_{c}$. The 


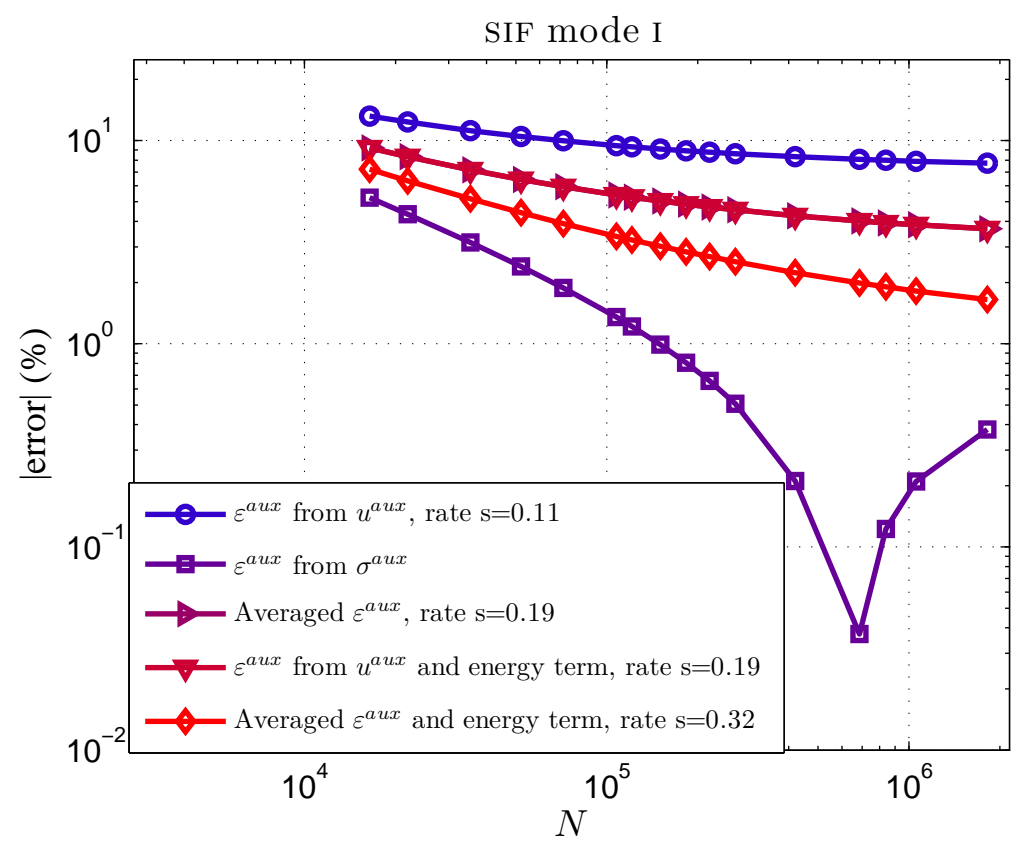

Figure 8. Results of the convergence study for the different interaction integral definitions for curved cracks. Convergence of the error in $K_{\mathrm{I}}$.

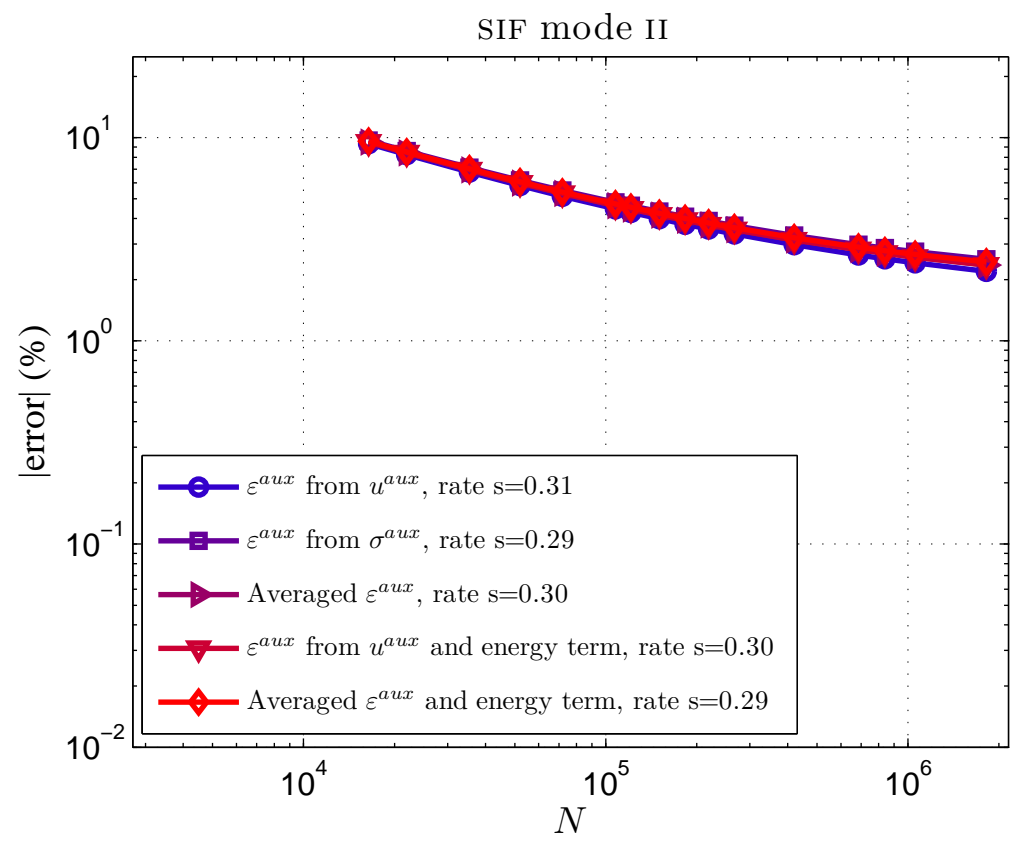

Figure 9. Results of the convergence study for the different interaction integral definitions for curved cracks. Convergence of the error in $K_{\mathrm{II}}$.

effect on the convergence rate for the error in $K_{\mathrm{I}}, K_{\mathrm{II}}$ and $J$ can be observed in Figures 10, 11 and 12 , respectively. It can be observed that the plateau function does not behave correctly with the interaction integral, showing a sign change for the error in $K_{\mathrm{I}}$ and a negative or almost zero 
convergence for the error in $K_{\mathrm{II}}$. However, both extraction functions show optimal convergence with the $J$-integral. This result is not surprising, because the ramp function leads to good behaviour with the interaction integral as it has no central region, thus controlling the effect of the approximations introduced by the auxiliary fields. The good results of the $J$-integral combined with the plateau function are explained because the $J$-integral does not involve auxiliary fields and the central region (plateau) avoids the contribution of the zone near the crack tip to the integral computation.

The bad behaviour of the plateau $q$-function in combination with the interaction integral can be explained as follows. When using the plateau function only a ring of elements contributes to the computation of the first part of the interaction integral (integral with $\partial_{j} q_{i}$ as factor). As these elements are far from the region where the auxiliary straight crack fields are acceptable, the contribution of this first integral is, in average, worse than when the ramp function is used. In addition, for the second integral (integral with $q_{i}$ as factor) most of the elements that lie between the extraction ring and the crack tip are multiplied by 1.0 (the plateau value). Since this second integral is not zero because we are using auxiliary fields for straight cracks, the undesired effect of this straight-crack auxiliary fields is increased. Therefore, convergence with the plateau function is worse than with the ramp function.

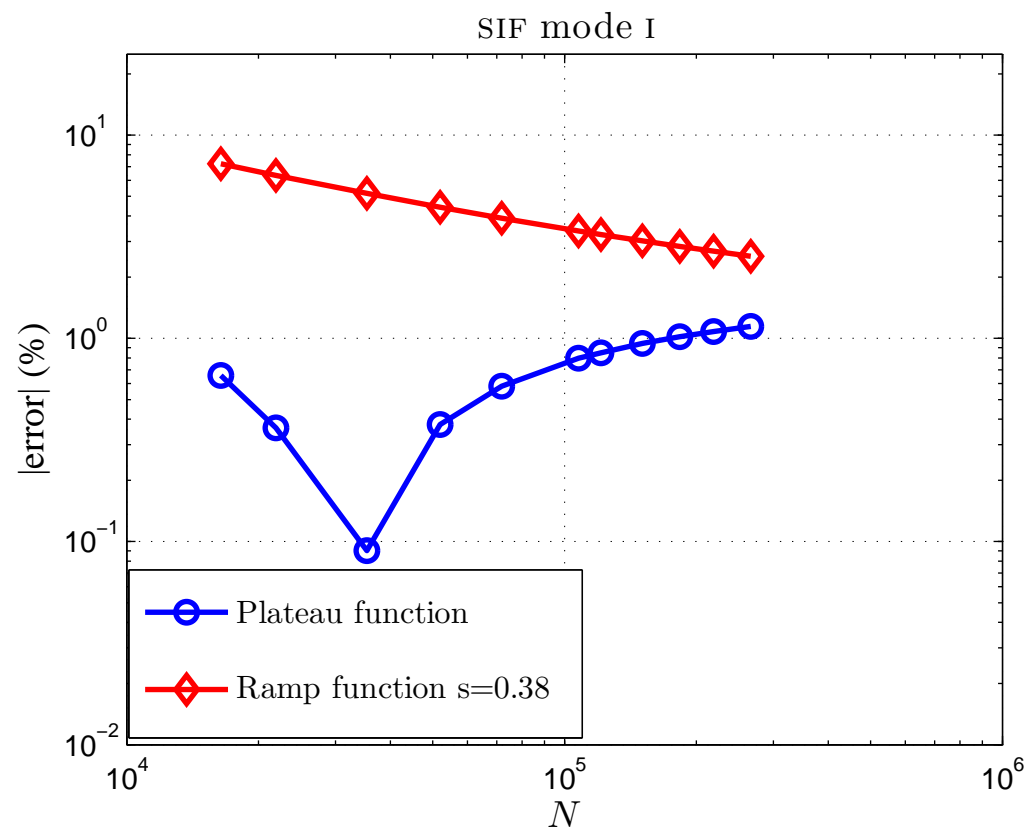

Figure 10. Effect of the extraction function $\alpha$ on the convergence. Convergence of the error in $K_{\mathrm{I}}$ using the interaction integral.

Finally, the influence of the size of the extraction domain is studied. The extraction domain radius $R$ is varied between $R=0.01 R_{c}$ and $R=0.9 R_{c}$. For the interaction integral, we use the expression that yields better results (averaged auxiliary strain field and the modified interaction integral expression 21). The ramp function is used in all cases. Fig. 13 presents the calculated convergence rate as a function of the extraction domain radius. Note that each point in Fig. 13 is obtained with a whole convergence study. In addition, the minimum error achieved in each of these 


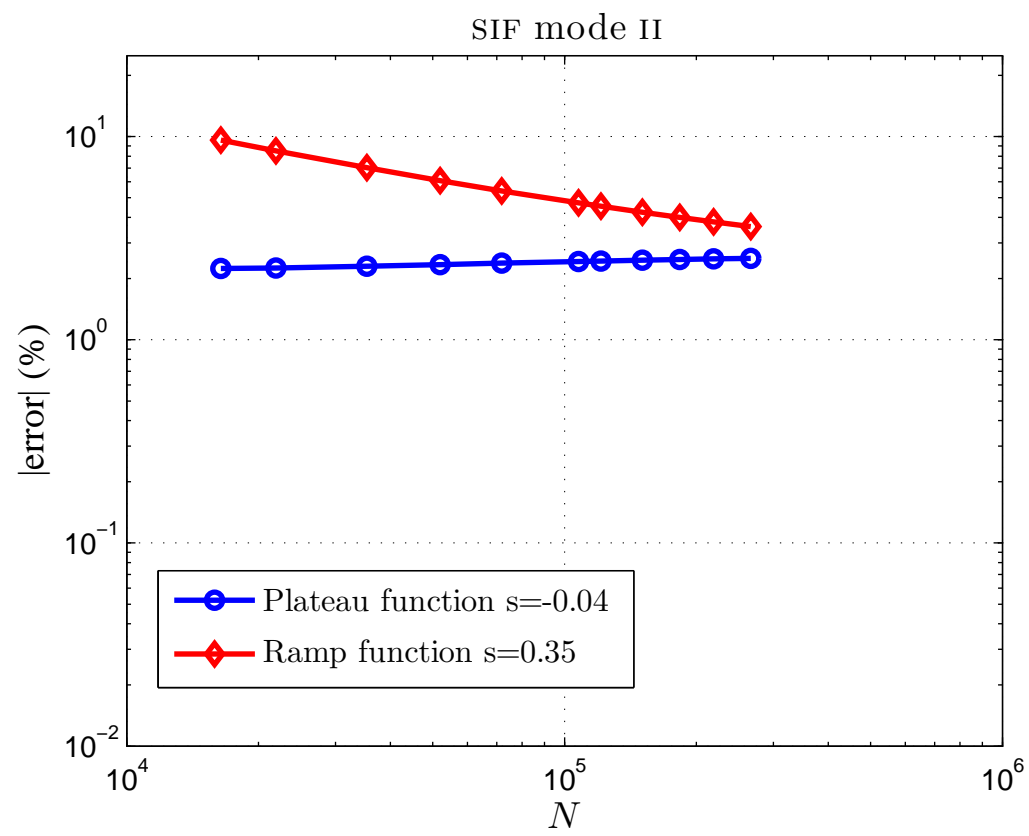

Figure 11. Effect of the extraction function $\alpha$ on the convergence. Convergence of the error in $K_{\mathrm{II}}$ using the interaction integral.

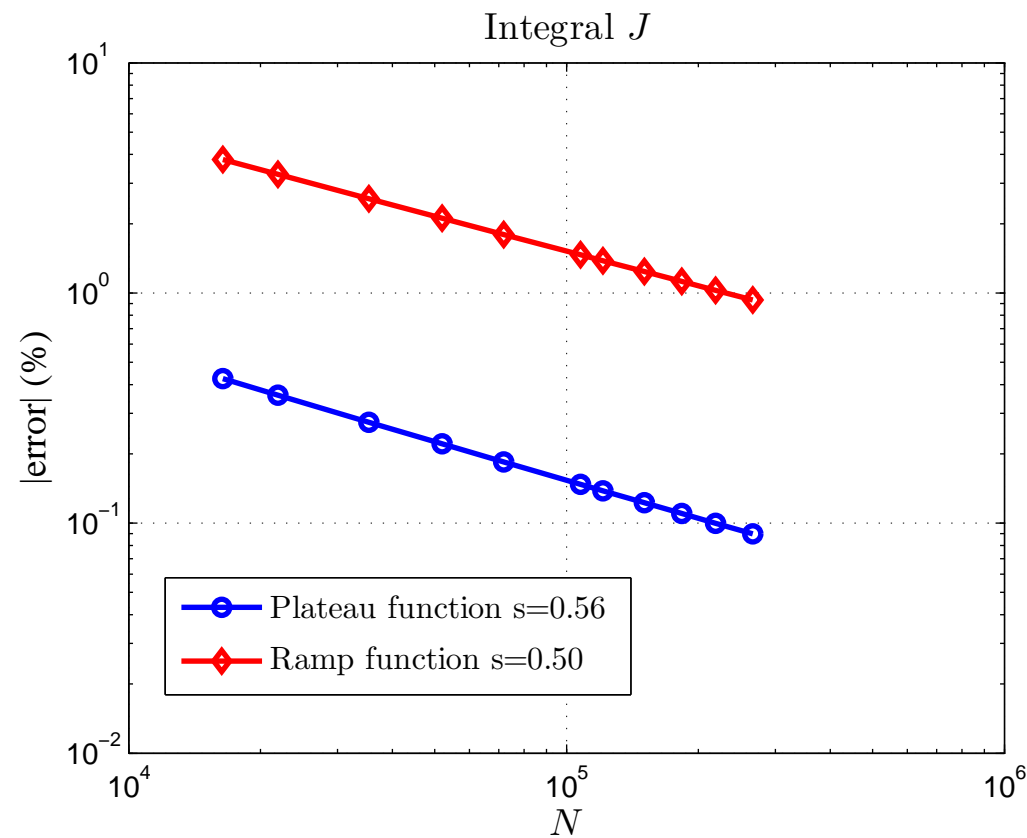

Figure 12. Effect of the extraction function $\alpha$ on the convergence. Convergence of the error in $J$ using the $J$-integral.

convergence studies is presented in Fig. 14. The results show that an extraction radius of about $10 \%$ of the curvature radius should be used in order to achieve good convergence rates. Note that a very small extraction radius would need a highly refined mesh in the vicinity of the crack tip. This is 
indicated in Fig. 14 by the loss of accuracy for very small radii. Note also that the convergence rates are close to the expected value of 0.5 for small radii (see Fig. 13). This suggests that the straight crack auxiliary fields are an acceptable approximation in this region.

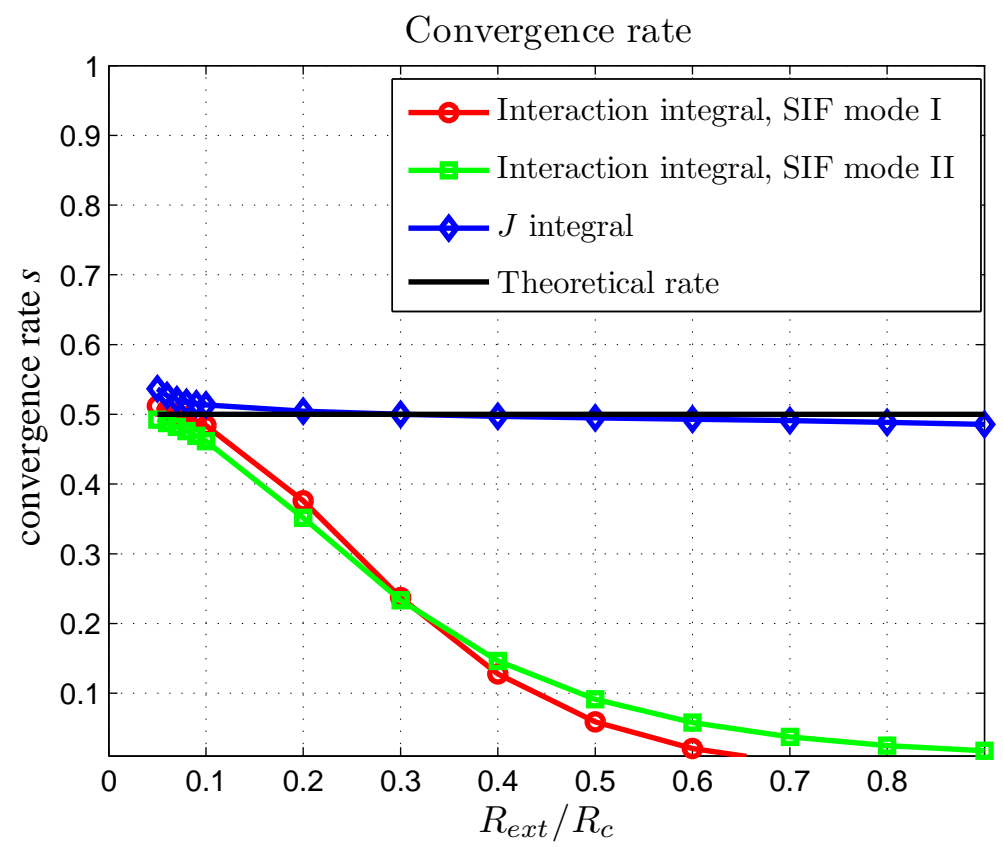

Figure 13. Convergence rates for the error in the SIFs and $J$ calculated with several extraction radii.

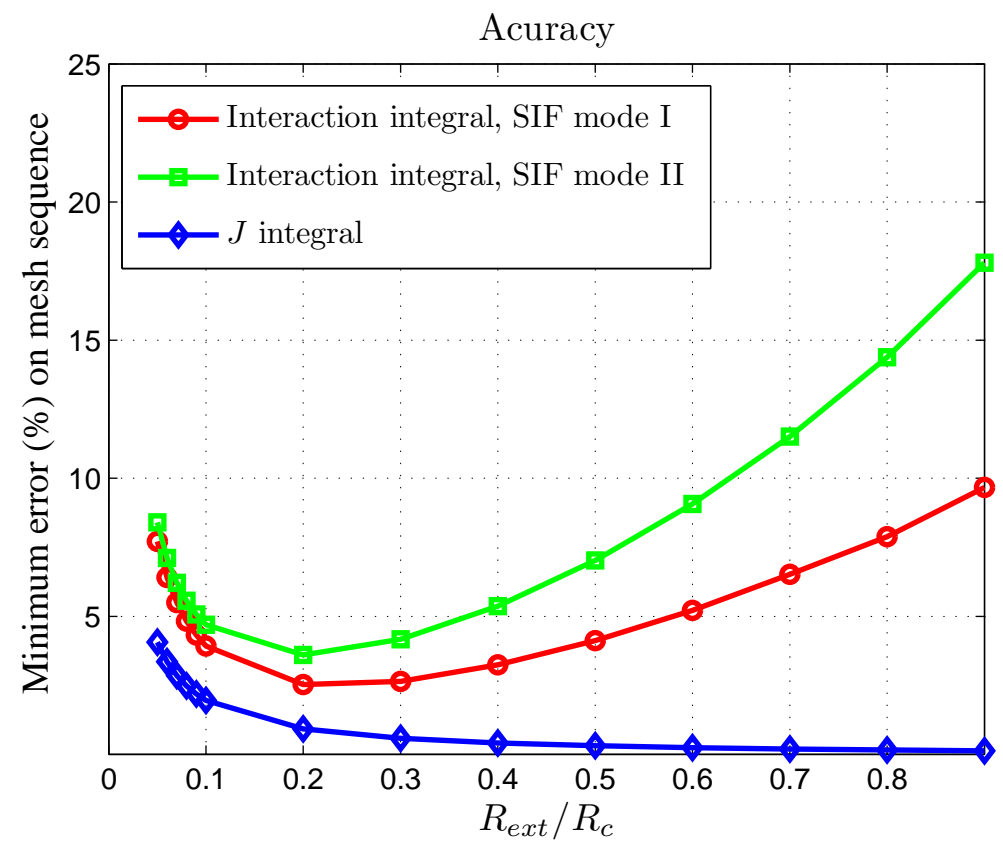

Figure 14. Minimum absolute error (\%) achieved in each of the convergence studies of Fig. 13. 
In order to verify the results, a last example is studied. The arc crack geometry with a subtended angle of $\beta=\pi / 4$ is analyzed using the options that led to the best results in the previous case $\beta=\pi / 2$. These options are: an orthogonal level set basis 6 , the modified interaction integral expression 21 (with an averaged auxiliary strain field), a ramp $q$-function 32 and an extraction domain of size $R / R_{c}=0.1$. The error convergence rates for $K_{\mathrm{I}}, K_{\mathrm{II}}$ and $J$-integral are shown in Fig. 15. The convergence rates are close to the optimum value and their behavior is similar to the $\beta=\pi / 2$ case. These results show that our proposal yields a good behaviour in terms of convergence rates.

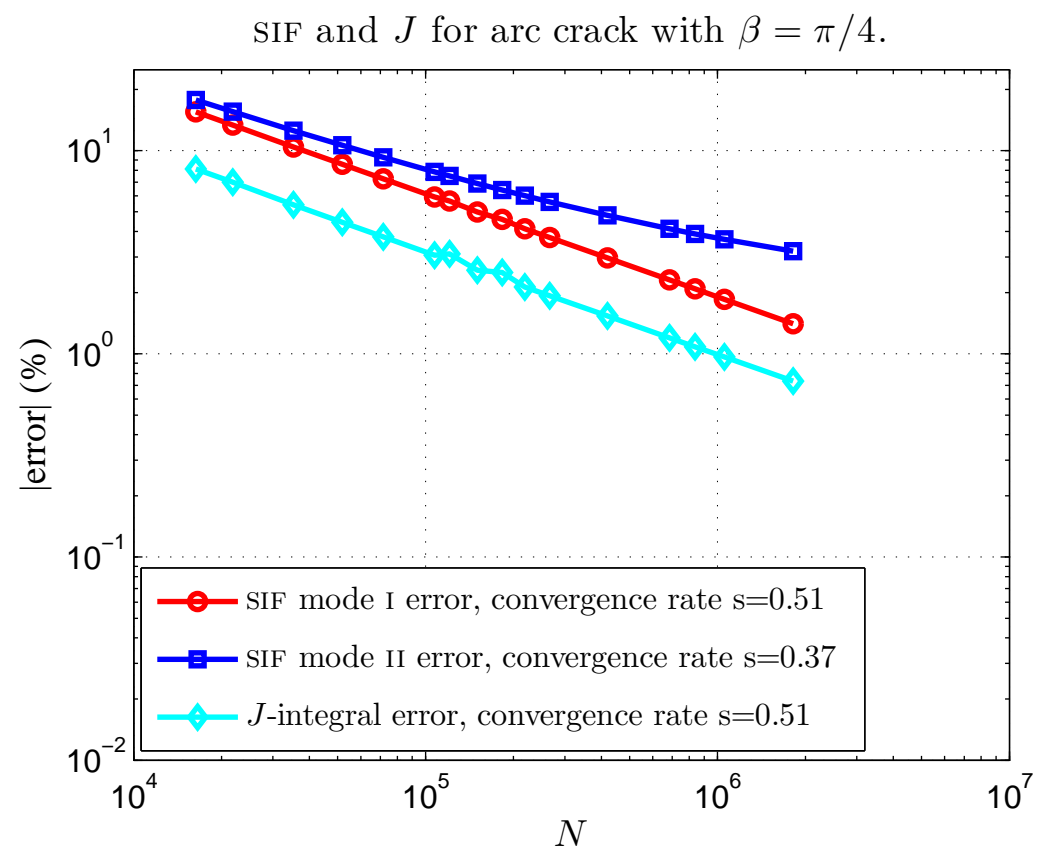

Figure 15. Arc crack with $\beta=\pi / 4$, verification of the results. Convergence rates for the error in the SIFs and $J$ with a ramp $q$-function and $R / R_{c}=0.1$.

\section{CONCLUSIONS}

Several conclusions can be drawn from the present study regarding the application of energy integrals to curved crack problems. The first is that the domain energy integrals exhibit good convergence rates when an orthogonal basis is used. If the basis is not orthogonal, then the optimum convergence rate cannot be assured. An orthogonal basis can be constructed, for example, using only the level set which describes the crack surface together with geometric considerations. When using this orthogonal basis, it has been shown that the convergence rate of the $J$-integral is the optimal for topological enrichment in XFEM.

If the interaction integral is used to extract the SIFs in a curved crack problem, its convergence to the exact solution cannot be assured, at least not with the optimal convergence rate. The reason is related to the definition of the auxiliary fields and the assumptions involved in the integral definition. 
We have shown that if the interaction integral is computed without introducing some simplifications related to the reciprocal relationship, an improvement in the accuracy is observed.

As far as the extraction function is concerned, this should be selected to control the influence of each term of the interaction integral expression. We can conclude that the use of a function whose derivative is not zero in the domain around the crack tip (ramp function) provides the best results with the interaction integral. However, the best accuracy with the $J$-integral is obtained with a plateau function but this function does not lead to good results with the interaction integral. In order to achieve a good convergence rate, a domain extraction size less than $10 \%$ of the curvature radius should be used. This also imposes some restrictions on the element size in the vicinity of the crack tip. Moreover, this introduces limitations on the use of geometrical XFEM enrichment in curved crack problems.

\section{ACKNOWLEDGEMENTS}

This work has been carried out within the framework of the research projects DPI2007-66995-C03-02 and DPI2010-20990 financed by the Ministerio de Economía y Competitividad. The support of the Generalitat Valenciana, Programme PROMETEO 2012/023 is also acknowledged.

\section{REFERENCES}

1. Moës N, Dolbow J, Belytschko T. A finite element method for crack growth without remeshing. International Journal for Numerical Methods in Engineering 1999;46:131-150.

2. Sukumar N, Moës N, Moran N, Belytschko T. Extended finite element method for three-dimensional crack modelling. International Journal for Numerical Methods in Engineering 2000;48:1549-1570.

3. Moës N, Gravouil A, Belytschko T. Non-planar 3D crack growth by the extended finite element and level sets-Part I: mechanical model. International Journal for Numerical Methods in Engineering 2002;53:2549-2568.

4. Gravouil A, Moës N,Belytschko T. Non-planar 3D crack growth by the extended finite element and level sets-Part II: Level set update International Journal for Numerical Methods in Engineering 2002;53:2569-2586.

5. Sukumar N, Prévost J-H. Modeling quasi-static crack growth with the extended finite element method. Part I: computer implementation. International Journal of Solids and Structures 2003;40(26):7513-7537.

6. Huang R, Sukumar N, Prévost J-H. Modeling quasi-static crack growth with the extended finite element method. Part II: numerical applications. International Journal of Solids and Structures 2003;40(26):7539-7552.

7. Rice JR. A path independent integral and the approximate analysis of strain concentration by notches and cracks. Journal of Applied Mechanics 1968;35:379-386.

8. Shih CF, Moran B, Nakamura T. Energy release rate along a three-dimensional crack front in a thermally stressed body. International Journal of Fracture 1986;30:79-102.

9. Moran B, Shih CF. Crack tip and associated domain integrals from momentum and energy balance. Engineering Fracture Mechanics 1987;27(6):615-642.

10. Walters M, Paulino G, Dodds R Jr. Interaction integral procedure for 3-D curved cracks including surface traction. Engineering Fracture Mechanics 2005;772:1635-1663.

11. Duflot M. A study of the representation of cracks with level sets. International Journal for Numerical Methods in Engineering 2006;70(11):1261-1302.

12. Sethian JA. Level Set Methods: evolving interfaces in geometry, fluid mechanics, computer vision and material sciences, Cambridge University Press, 1996.

13. Osher S, Fedkiw RP. Level Set Methods: an overview and some recent results. Journal of Computational Physics 2001;169:463-502.

14. Nahta R, Moran B. Domain integrals for axisymmetric interface crack problems. International Journal of Solids and Structures 1993;30(15):2027-2040.

15. Gosz M, Dolbow J, Moran B. Domain integral formulation for stress intensity factor computation along curved three-dimensional interface cracks. International Journal of Solids and Structures 1998;35(15):1763-1783. 
16. Gosz M, Moran B. An interaction energy integral method for computation of mixed-mode stress intensity factors along non-planar crack fronts in three dimensions. Engineering Fracture Mechanics 2002;69:299-319.

17. Eriksson K. A general expression for an area integral of a point-wise $J$ for curved cracks. International Journal of Fracture 2000;106:65-80.

18. Sukumar N. Element partitioning code in 2-D and 3-D for the extended finite element method. http://dilbert.engr.ucdavis.edu/ suku/xfem, 2000.

19. Laborde P, Pommier J, Renard Y, Salaün M. High-order extended finite element method for cracked domains. International Journal for Numerical Methods in Engineering 2005;64:354-381.

20. Eshelby JD. The elastic energy-momentum tensor. Journal of Elasticity 1975;5(3-4):321-335.

21. Muskhelishvili NI. Some basic problems in the mathematical theory of elasticity. Leiden: Noordhoff; 1952.

22. Simpson RN, Trevelyn J, Evaluation of $J_{1}$ and $J_{2}$ integrals for curved cracks using an enriched boundary element method. Engineering Fracture Mechanics 2011;78:623-637.

23. Cheng KW, Fries TP. Higher-order XFEM for curved strong and weak discontinuities, International Journal for Numerical Methods in Engineering 2010;82:564-590.

24. Tarancón JE, Vercher A, Giner E, Fuenmayor FJ. Enhanced blending elements for XFEM applied to linear elastic fracture mechanics. International Journal for Numerical Methods in Engineering 2009;77:126-148.

25. Béchet E, Minnebo H, Moës N, Burgardt B. Improved implementation and robustness study of the X-FEM fortress analysis around cracks. International Journal for Numerical Methods in Engineering 2005;64:1033-1056.

26. Stazi FL, Budyn E, Chessa J, Belytschko T. An extended finite element method with higher-order elements for curved cracks. Computational Mechanics 2003;31:38-48.

27. Chessa J, Wang H, Belytschko T. On the construction of blending elements for local partition of unity enriched finite elements. International Journal for Numerical Methods in Engineering 2003;57(7):1015-1038. 